\title{
Dynamics of an Information Spreading Model with Isolation
}

\author{
Xia-Xia Zhao ${ }^{1,2}$ and Jian-Zhong Wang ${ }^{1,2}$ \\ ${ }^{1}$ National Key Laboratory for Electronic Measurement Technology, North University of China, Taiyuan, Shanxi 030051, China \\ ${ }^{2}$ Key Laboratory of Instrumentation Science and Dynamic Measurement, Ministry of Education, North University of China, \\ Taiyuan, Shanxi 030051, China
}

Correspondence should be addressed to Xia-Xia Zhao; xiaxiazhao12@163.com

Received 20 March 2014; Accepted 4 April 2014; Published 23 April 2014

Academic Editor: Sanling Yuan

Copyright (c) 2014 X.-X. Zhao and J.-Z. Wang. This is an open access article distributed under the Creative Commons Attribution License, which permits unrestricted use, distribution, and reproduction in any medium, provided the original work is properly cited.

Information plays an important role in modern society. In this paper, we presented a mathematical model of information spreading with isolation. It was found that such a model has rich dynamics including Hopf bifurcation. The results showed that, for a wide range of parameters, there is a bistable phenomenon in the process of information spreading and thus the information cannot be well controlled. Moreover, the model has a limit cycle which implies that the information exhibits periodic outbreak which is consistent with the observations in the real world.

\section{Introduction}

In recent years, information plays a more and more important role in the real world. For a decision maker, if he does not have reasonable information, he will make a wrong decision which may cause bad consequences. However, long time series of information are hard to obtain and thus it may provide useful information by constructing mathematical models. As a result, modeling the process of information spreading has been one of the central themes in the field of science $[1,2]$.

Information can be divided into many types. For some useful information such as advanced culture and useful knowledge, it is encouraged to develop both widely and deeply. However, there is also some bad information and the typical one is rumor [3-8]. Rumor is a social phenomenon accompanying the development of society which may bring some corrosive social effects. For example, in 2007, some people circulated false news that the bananas from Hainan were toxic which caused huge economic loss [9]. In this sense, we need to isolate some information by taking control measures.

There is some work on information spreading by using mathematical models. Based on SIS models, Leskovec et al. presented a cascading information model and found how blogs behaved and how information propagated through the blogosphere [10]. Gruhl et al. posed a SIRS model to calculate the spread rate of information between nodes [11]. Narayanam and Narahari proposed a linear threshold model to describe information spreading [12]. Moreover, some scholars used dynamical models [13] and statistical analysis [14] to investigate the propagation of information.

In this paper, we aim to present a mathematical model to describe the information spreading with isolation. What is more, we want to reveal its dynamical behavior by both mathematical analysis and numerical results. The paper is organized as follows. In Section 2, we construct an information spreading model by using three ordinary differential equations (ODES). In Section 3, we show that this model has rich dynamics. Finally, conclusions and discussions are presented in Section 4.

\section{A Mathematical Model}

To well describe the model, we firstly give four main assumptions, which are as follows.

(i) There are three kinds of individuals: the population who has no information $(X)$, the population who has information $(Y)$, and the population who is isolated $(Z)$ by isolation.

(ii) There are birth and death events and all the newborn has no information. 


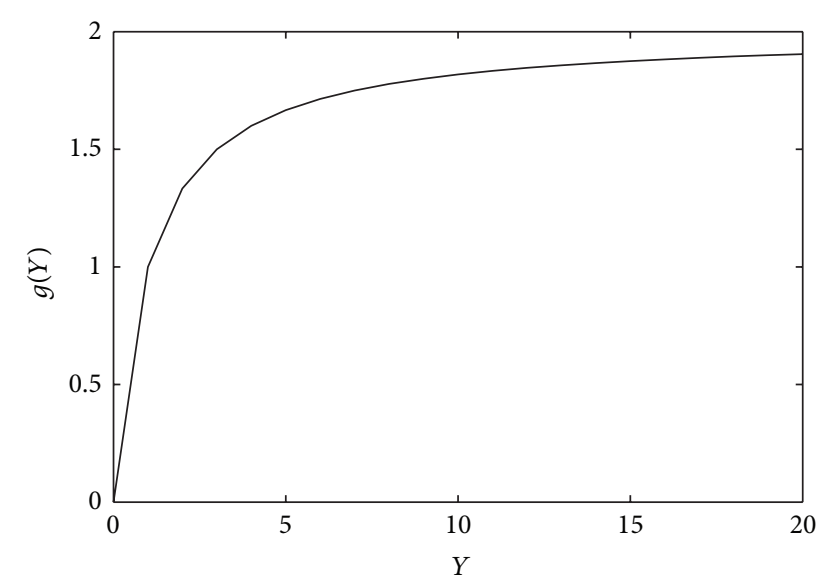

Figure 1: Plot of $g(Y)$ as a function of $Y$ with $a=2$ and $b=1$ (color online).

(iii) The spread rate of information is

$$
g(Y)=\frac{a Y}{(1+b Y)}
$$

which has a saturation effect (cf. Figure 1).

(iv) Since information spreading has stages, isolation term may have different forms at different stages of information spreading. We choose isolation term having the following form:

$$
I(Y)=\frac{c_{1}+c_{2} Y}{1+Y} Y
$$

For the small number of $Y, I(Y) \rightarrow c_{1} Y$ and $I(Y) \rightarrow c_{2} Y$ for large number of $Y$.

On the basis of the above assumptions, we have the following model:

$$
\begin{gathered}
\frac{d X}{d t}=A-d X-g(Y) X, \\
\frac{d Y}{d t}=g(Y) X-d Y-I(Y), \\
\frac{d Z}{d t}=I(Y)-d Z,
\end{gathered}
$$

where $A$ is the birth rate of the population and $d$ is the natural death rate of the population. Since the first two equations in (3a), (3b), and (3c) are independent of the variable $Z$, it suffices to consider the following reduced model:

$$
\begin{gathered}
\frac{d X}{d t}=A-d X-\frac{a X Y}{1+b Y}=h_{1}(X, Y), \\
\frac{d Y}{d t}=\frac{a X Y}{1+b Y}-d Y-\frac{c_{1}+c_{2} Y}{1+Y} Y=h_{2}(X, Y) .
\end{gathered}
$$

\section{Dynamical Behaviors}

The first step in analyzing the model (4a) and (4b) is to determine equilibria points by solving the following equations:

$$
\begin{gathered}
A-d X-\frac{a X Y}{1+b Y}=0, \\
\frac{a X Y}{1+b Y}-d Y-\frac{c_{1}+c_{2} Y}{1+Y} Y=0 .
\end{gathered}
$$

System (4a) and (4b) always has an equilibrium $E_{0}=$ $(A / d, 0)$ corresponding to the case that there is no information. The Jacobian matrix of the linearization of system (4a) and (4b) at this equilibrium is [15]

$$
\mathbf{J}=\left(\begin{array}{cc}
-d & -a \frac{A}{d} \\
0 & a \frac{A}{d}-\left(d+c_{1}\right)
\end{array}\right),
$$

which has negative eigenvalues, implying the asymptotic stability of this equilibrium if and only if

$$
A a<d\left(d+c_{1}\right) .
$$

Denote

$$
S_{1}=\frac{A a}{d\left(d+c_{1}\right)} .
$$

If $S_{1}<1$, then there is no information; otherwise the information will spread.

In order to obtain positive solutions of (5a) and (5b), we eliminate $X$ using the first equation of (5a) and (5b) and substitute it into the second equation to give an equation of the form

$$
P_{1} Y^{2}+P_{2} Y+P_{3}=0
$$

where

$$
\begin{gathered}
P_{1}=b d^{2}+b d c_{2}+a d+a c_{2}, \\
P_{2}=b d c_{1}+b d^{2}+a d+d^{2}+a c_{1}-A a+d c_{2}, \\
P_{3}=d^{2}+d c_{1}-A a .
\end{gathered}
$$

It should be noted that $P_{3}>0(<0$ or $=0)$ if and only if $S_{1}>1(<1$ or $=1)$. That is to say, there is a unique nonzero solution of (9) denoted as $Y=-P_{2} / P_{1}$ if $S_{1}=1$ and it is positive if and only if $P_{2}<0$. Since equilibria depend continuously on $S_{1}$, there is an interval to the left of $S_{1}=1$ on which there are two positive equilibria and

$$
Y_{1,2}=\frac{-P_{2} \pm \sqrt{P_{2}^{2}-4 P_{3}}}{2 P_{1}} .
$$

As a result, we can conclude that system (4a) and (4b) has a backward bifurcation at $S_{1}=1$ if and only if $P_{2}<0[16,17]$.

We can also give an explicit criterion of $c_{2}$ in terms of other parameters for the existence of a backward bifurcation at $S_{1}=1$ equals $d^{2}+d c_{1}=A a . P_{2}<0$ implies that

$$
b d c_{1}+b d^{2}+a d+d^{2}+a c_{1}+d c_{2}<A a,
$$


and thus

$$
b d c_{1}+b d^{2}+a d+d^{2}+a c_{1}+d c_{2}<d^{2}+d c_{1},
$$

which equals

$$
c_{2}<\frac{d c_{1}-b d c_{1}-b d^{2}-a d-a c_{1}}{d} \triangleq c_{2}^{*} .
$$

If (14) is satisfied, there is a backward bifurcation at $S_{1}=$ 1. Then, there are two positive equilibria for an interval of values of $S$ from a value $S_{2}$ to $S_{1}=1$. To calculate $S_{2}$, we use $P_{2}^{2}-4 P_{1} P_{3}=0$ to get a quadratic equation of parameter $A$, which has the following form:

$$
\begin{aligned}
& a^{2} A^{2} \\
& +2 A a\left(b d^{2}+2 b d c_{2}+a d+2 a c_{2}-b d c_{1}-d^{2}-a c_{1}-d c_{2}\right) \\
& +\left(-d c_{2}+b d c_{1}-d^{2}+a d+a c_{1}+b d^{2}\right)^{2}=0 .
\end{aligned}
$$

Note that $P_{2}<0$ and $S_{1}<1$, which equals

$$
\frac{b d c_{1}+b d^{2}+a d+d^{2}+a c_{1}+d c_{2}}{a}<A<\frac{d\left(d+c_{1}\right)}{a} .
$$

In that case, we have that

$$
\begin{aligned}
A_{c}= & \left(d^{2}-a d-2 a c_{2}+a c_{1}-b d^{2}+d c_{2}-2 b d c_{2}\right. \\
& \left.+b d c_{1}+2 \sqrt{(a-d+b d)(a+b d)\left(d+c_{2}\right)\left(c_{2}-c_{1}\right)}\right) \\
& \times a^{-1} .
\end{aligned}
$$

As a result, the expression of $S_{2}$

$$
S_{2}=\frac{A_{c} a}{d\left(d+c_{1}\right)} .
$$

We fix $d=0.1, a=0.001, b=0.01, c_{1}=1$, and $c_{2}=0.5$ and show that system (4a) and (4b) has a backward bifurcation in Figure 2.

In the following, we discuss the case when $S_{1}<1$. If system (4a) and (4b) has a positive solution, the following condition must be satisfied:

$$
P_{2}<0, \quad \Delta \geq 0 .
$$

Note that $P_{2}<0$ equals $b d c_{1}+b d^{2}+a d+d^{2}+a c_{1}+d c_{2}<$ $A a$. And $S_{1}<1$ equals $A a<d\left(d+c_{1}\right)$. As a result, we have $b d c_{1}+b d^{2}+a d+d^{2}+a c_{1}+d c_{2}<d\left(d+c_{1}\right)$ which leads to $a+b d<d$.

The discussions above yield the following results.

(I) For the case $c_{1} \leq c_{2}, E_{0}$ is always a boundary equilibrium of system (4a) and (4b) and $E^{*}$ is the unique positive equilibria of system (4a) and (4b) if and only if $S_{1}>1$.

(II) For the case $c_{1}>c_{2}, E_{0}$ is always a boundary equilibrium of system (4a) and (4b) and system (4a) and (4b)

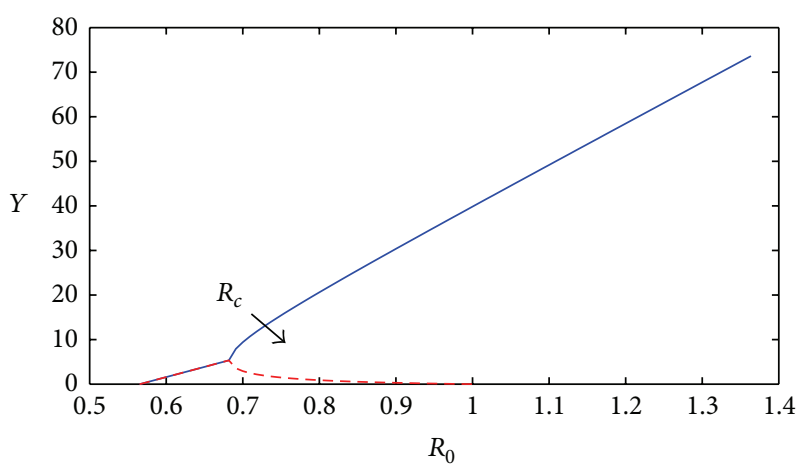

Figure 2: The sizes of population $Y$ at equilibria versus $S_{1}$ with $d=$ $0.1, a=0.001, b=0.01, c_{1}=1$, and $c_{2}=0.5$ (color online).

has a unique positive equilibrium if $S_{1}>1$. Furthermore, system (4a) and (4b) has two positive equilibria $E_{1}$ and $E_{2}$ if $S_{2}<S_{1}<1$ and $a+b d<d$.

In the following, we aim to investigate the global stability of the equilibrium by considering two cases: (i) $c_{1} \leq c_{2}$ and (ii) $c_{1}>c_{2}$.

For the first case, we can obtain the Jacobian matrix of system (4a) and (4b) at equilibrium $(X, Y)$ is

$$
J=\left(\begin{array}{cc}
-d-\frac{a Y}{1+b Y} & -\frac{a X}{(1+b Y)^{2}} \\
\frac{a Y}{1+b Y} & \frac{a X}{(1+b Y)^{2}}-d-\frac{c_{1}+2 c_{2} Y+c_{2} Y^{2}}{(1+Y)^{2}}
\end{array}\right) .
$$

By direct calculations, we can easily obtain det $J\left(E_{0}\right)=-A a+$ $d\left(d+c_{1}\right)>0, \operatorname{tr} J\left(E_{0}\right)=a A / d-2 d-c_{1}<0$ if $S_{1}<1$. And hence all the eigenvalues of characteristic equation of $E_{0}$ have negative real parts, which means that the equilibrium $E_{0}$ is locally asymptotically stable.

Let

$$
\Omega=\left\{(X, Y) \mid X, Y \geq 0, X+Y \leq \frac{A}{d}\right\} .
$$

By (4a) and (4b), we have

$$
\left.\frac{d X}{d t}\right|_{E_{0}}=A>0,\left.\quad \frac{d Y}{d t}\right|_{E_{0}}=0 .
$$

Denote $N_{1}(t)=X(t)+Y(t)$; then

$$
\left.\frac{d N_{1}}{d t}\right|_{N_{1}=A / d} \leq A-\left.d N_{1}\right|_{N_{1}=A / d}=0 .
$$

Hence, $\Omega$ is a positively invariant set of $(4 \mathrm{a})$ and $(4 \mathrm{~b})$ and attracts all the positive orbits of (4a) and (4b) state in $R_{2}^{+}$, which means that the equilibrium $E_{0}$ is globally asymptotically stable if $S_{1}<1$.

It is easy to obtain that $E^{*}$ is locally stable when $S_{1}>1$. Then, we use Dulac theorem to exclude the limit cycle [18].

Take the Dulac function

$$
D=\frac{1}{X Y}
$$




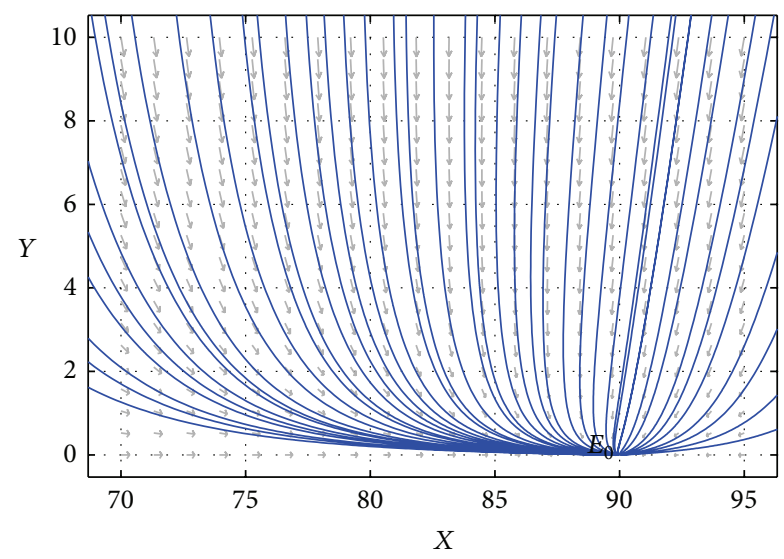

Figure 3: Phase plane of $X-Y$ with $A=9, d=0.1, a=0.001$, $b=0.01, c_{1}=0.1$, and $c_{2}=0.5$ (color online).

Then, we have

$$
\frac{\partial\left(h_{1} D\right)}{\partial X}+\frac{\partial\left(h_{2} D\right)}{\partial Y}=-\frac{A}{X^{2} Y}-\frac{a b}{(1+Y)^{2}}-\frac{c_{1}-c_{2}}{X(1+Y)^{2}}<0,
$$

which means that system (4a) and (4b) has no limit cycles and the positive equilibrium is globally asymptotically stable.

As a result, we have the following conclusions.

(i) The equilibrium $E_{0}$ is globally asymptotically stable if $S_{1}<1$ and unstable if $S_{1}>1$.

(ii) When $S_{1}>1$, the unique endemic equilibrium is globally asymptotically stable.

In Figure 3, we fixed $A=9, d=0.1, a=0.001, b=0.01$, $c_{1}=0.1$, and $c_{2}=0.5$ which satisfies $S_{1}<1$. It can be found from this figure that $E_{0}$ is globally asymptotically stable.

In Figure 4, we fixed $A=90, d=0.1, a=0.001, b=$ $0.01, c_{1}=0.1$, and $c_{2}=0.5$ which satisfies $S_{1}>1$. It can be found from this figure that $E_{0}$ is not stable and the unique endemic equilibrium is globally asymptotically stable.

For the second case $c_{1}>c_{2}$, we need to discuss three situations:
(I) $S_{1}<S_{2}$;
(II) $S_{1}=S_{2}$;
(III) $S_{2}<S_{1}<1$;
(IV) $S_{1}>1$.

Since dynamical behaviors of system (4a) and (4b) in situation (I) are simple, we will investigate the later three cases.

If $S_{1}=S_{2}$, system (4a) and (4b) has a unique positive equilibrium $E_{c}\left(X_{c}, Y_{c}\right)$, where

$$
\begin{gathered}
X_{c}=\left[\frac{d}{a}+\frac{c_{1}+c_{2} Y_{c}}{a\left(1+Y_{c}\right)}\right]\left(1+b Y_{c}\right), \\
Y_{c}=-\frac{b d c_{1}+b d^{2}+a d+d^{2}+a c_{1}-A a+d c_{2}}{2\left(b d^{2}+b d c_{2}+a d+a c_{2}\right)} .
\end{gathered}
$$

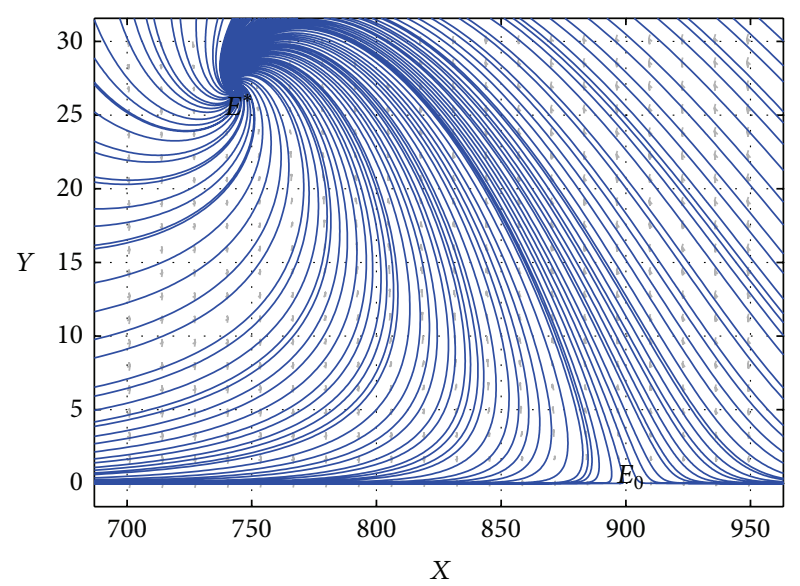

Figure 4: Phase plane of $X-Y$ with $A=90, d=0.1, a=0.001$, $b=0.01, c_{1}=0.1$, and $c_{2}=0.5$ (color online).

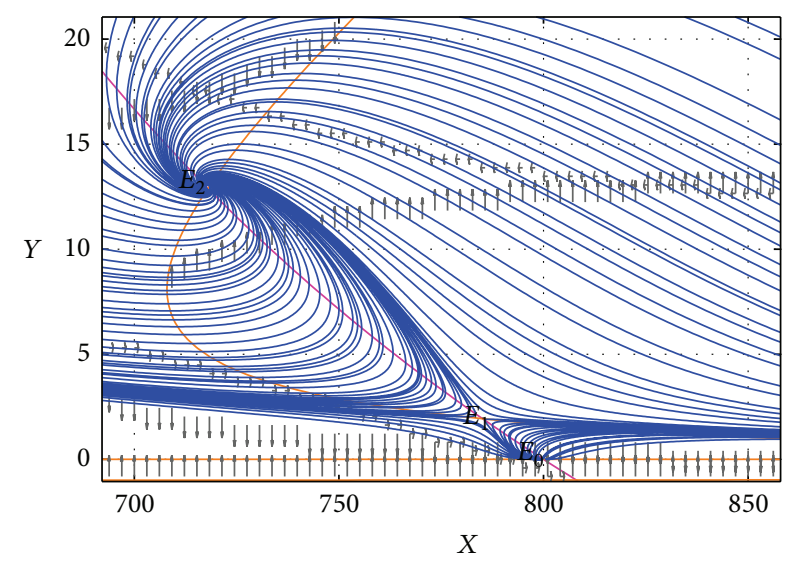

Figure 5: Phase plane of $X-Y$ with $A=80, d=0.1, a=0.001$, $b=0.01, c_{1}=1$, and $c_{2}=0.5$ (color online).

By calculations, one can find that $\operatorname{det} J_{E_{c}}=\operatorname{tr} J_{E_{c}}=0$, which means that Jacobian matrix has a zero eigenvalue with multiplicity 2 . In other words, the positive equilibrium $E_{c}$ of system (4a) and (4b) is a cusp of codimension 2 [19].

If $S_{2}<S_{1}<1$, then system (4a) and (4b) exhibits multiple positive equilibria. In such a case, it has been shown that a stable positive equilibrium coexists with the stable boundary equilibrium $\left(E_{0}\right)$, which is named as bistable phenomenon.

In Figure 5, we fixed $A=80, d=0.1, a=0.001, b=0.01$, $c_{1}=1$, and $c_{2}=0.5$ which satisfies $S_{2}<S_{1}<1$. We can see from this figure that $E_{0}$ and $E_{2}$ are stable. In this case, the information cannot be well controlled.

We assume that $S_{1}>1$. If $\operatorname{tr}(\mathbf{J})<0$, then the positive equilibrium $E_{*}$ of system (4a) and (4b) is a stable node or focus; if $\operatorname{tr}(J)>0$, then $E_{*}$ is unstable and system (4a) and (4b) has at least one limit cycle; if $\operatorname{tr}(J)=0$, then $E_{*}$ is a center.

In Figure 6, we fixed $A=150, d=0.1, a=0.001, b=$ $0.01, c_{1}=1$, and $c_{2}=0.5$ which satisfies $S_{1}>1$. We can see from this figure that $E_{*}$ is unstable and system (4a) and (4b) has a stable limit cycle in which Hopf bifurcation occurs in system (4a) and (4b). These results show that the information 


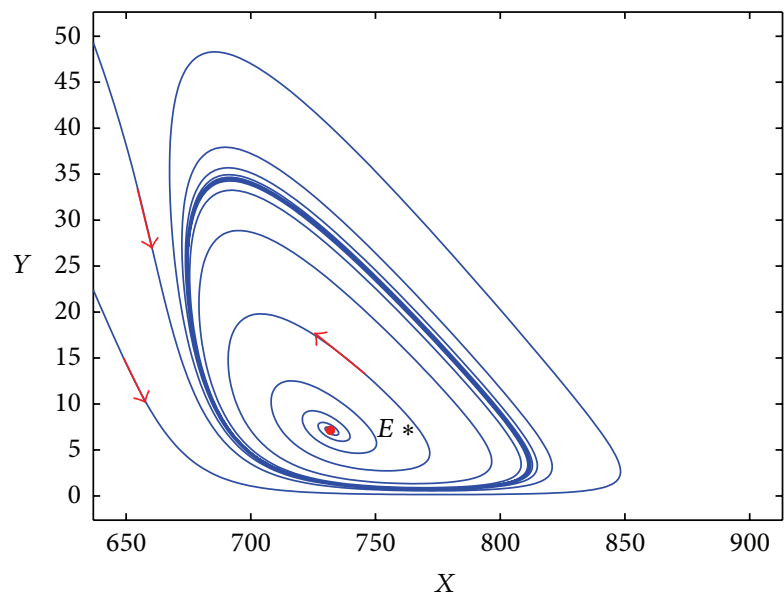

FIgURE 6: Phase plane of $X-Y$ with $A=150, d=0.1, a=0.001$, $b=0.01, c_{1}=1$, and $c_{2}=0.5$ (color online).

exhibits periodic outbreak which is consistent with the real data [20].

\section{Discussion and Conclusion}

Information has a great effect on social network, which is a double-edged sword. In this paper, we investigated an information spreading model with isolation. For the dynamical model, we obtained the global stability of the equilibria and bifurcation behaviors, based on both mathematical analysis and numerical results. In a word, information spreading model can have rich dynamics which may provide some new insights for policy decisions on information.

It should be noted that our results are based on the assumption of a homogeneously mixing population. That is to say, we assume that contacts between different individuals are in the same form. This assumption ignores individual differences and thus is not reasonable [21-24]. As a result, we need to investigate the information spreading model in social network in further research.

\section{Conflict of Interests}

The authors declare that there is no conflict of interests regarding the publication of this paper.

\section{References}

[1] L. K. Gallos, R. Cohen, P. Argyrakis, A. Bunde, and S. Havlin, "Stability and topology of scale-free networks under attack and defense strategies," Physical Review Letters, vol. 94, no. 18, Article ID 188701, 2005.

[2] Y. Moreno, M. Nekovee, and A. F. Pacheco, "Dynamics of rumor spreading in complex networks," Physical Review E. Statistical, Nonlinear, and Soft Matter Physics, vol. 69, no. 6, Article ID 066130, p. 1, 2004.

[3] D. J. Daley and D. G. Kendall, "Epidemics and Rumours," Nature, vol. 204, no. 4963, article 1118, 1964.
[4] D. H. Zanette, "Critical behavior of propagation on small-world networks," Physical Review E. Statistical, Nonlinear, and Soft Matter Physics, vol. 64, no. 5, Article ID 050901, 2001.

[5] L. Lü, D.-B. Chen, and T. Zhou, "The small world yields the most effective information spreading," New Journal of Physics, vol. 13, Article ID 123005, 2011.

[6] A. L. Hill, D. G. Rand, M. A. Nowak, and N. A. Christakis, "Infectious disease modeling of social contagion in networks," PLoS Computational Biology, vol. 6, no. 11, Article ID e1000968, 2010.

[7] T. House, "Modelling behavioural contagion," Journal of the Royal Society Interface, vol. 8, no. 59, pp. 909-912, 2011.

[8] Z.-Q. Zhu, C.-J. Liu, J.-L. Wu, J. Xu, and B. Liu, "The influence of human heterogeneity to information spreading," Journal of Statistical Physics, vol. 154, no. 6, pp. 1569-1577, 2014.

[9] http://www.iyaxin.com/content/2008-10/21/content_450073 .htm.

[10] J. Leskovec, M. McGlohon, C. Faloutsos, N. Glance, and M. Hurst, "Patterns of cascading behavior in large blog graphs," in Proceedings of the 7th SIAM International Conference on Data Mining, pp. 551-556, April 2007.

[11] D. Gruhl, D. Liben-Nowell, R. Guha, and A. Tomkins, "Information diffusion through blogspace," in Proceedings of the 13th International World Wide Web Conference Proceedings (WWW '04), pp. 491-501, May 2004.

[12] R. Narayanam and Y. Narahari, "A shapley value-based approach to discover influential nodes in social networks," IEEE Transactions on Automation Science and Engineering, vol. 8, no. 1, pp. 130-147, 2011.

[13] M. Lahiri and M. Cebrian, "The genetic algorithm as a general diffusion model for social networks," in Proceedings of the 24th AAAI Conference on Artificial Intelligence, pp. 494-499, July 2010.

[14] B. Suh, L. Hong, P. Pirolli, and E. H. Chi, "Want to be retweeted? Large scale analytics on factors impacting retweet in twitter network," in Proceedings of the 2nd IEEE International Conference on Social Computing (SocialCom '10), pp. 177-184, August 2010.

[15] J. Guckenheimer and P. Holmes, Nonlinear Oscillations, Dynamical Systems, and Bifurcations of Vector Fields, vol. 42, Springer, New York, NY, USA, 1990.

[16] J. Arino, C. C. McCluskey, and P. van den Driessche, "Global results for an epidemic model with vaccination that exhibits backward bifurcation," SIAM Journal on Applied Mathematics, vol. 64, no. 1, pp. 260-276, 2003.

[17] J. Dushoff, W. Huang, and C. Castillo-Chavez, "Backwards bifurcations and catastrophe in simple models of fatal diseases," Journal of Mathematical Biology, vol. 36, no. 3, pp. 227-248, 1998.

[18] L. Perko, Differential Equations and Dynamical Systems, vol. 7, Springer, New York, NY, USA, 2nd edition, 1996.

[19] Y. A. Kuznetsov, Elements of Applied Bifurcation Theory, vol. 112, Springer, New York, NY, USA, 1995.

[20] J. V. Carlis and J. A. Konstan, "Interactive visualization of serial periodic data," in Proceedings of the 11th Annual ACM Symposium on User Interface Software and Technology (UIST '98), pp. 29-38, November 1998.

[21] M. Yang, G. Chen, and X. Fu, "A modified SIS model with an infective medium on complex networks and its global stability," Physica A. Statistical Mechanics and its Applications, vol. 390, no. 12, pp. 2408-2413, 2011. 
[22] A.-L. Barabási and R. Albert, "Emergence of scaling in random networks," Science, vol. 286, no. 5439, pp. 509-512, 1999.

[23] A.-L. Barabási, R. Albert, and H. Jeong, "Mean-field theory for scale-free random networks," Physica A. Statistical Mechanics and its Applications, vol. 272, no. 1, pp. 173-187, 1999.

[24] R. Pastor-Satorras and A. Vespignani, "Epidemic spreading in scale-free networks," Physical Review Letters, vol. 86, no. 14, pp. 3200-3203, 2001. 


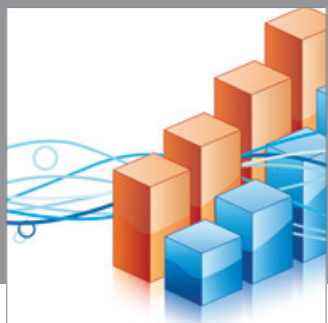

Advances in

Operations Research

mansans

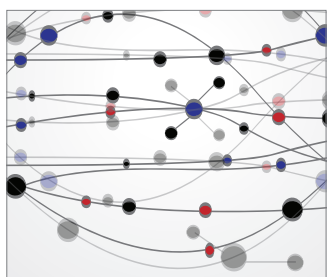

The Scientific World Journal
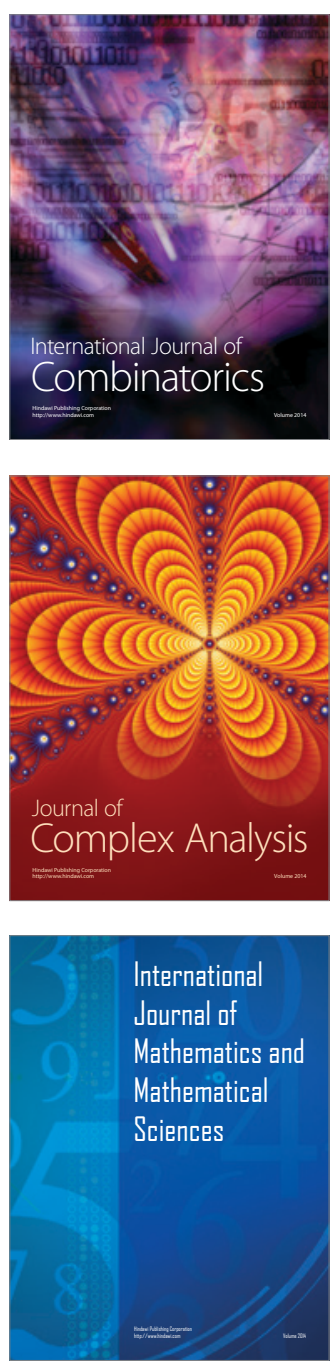
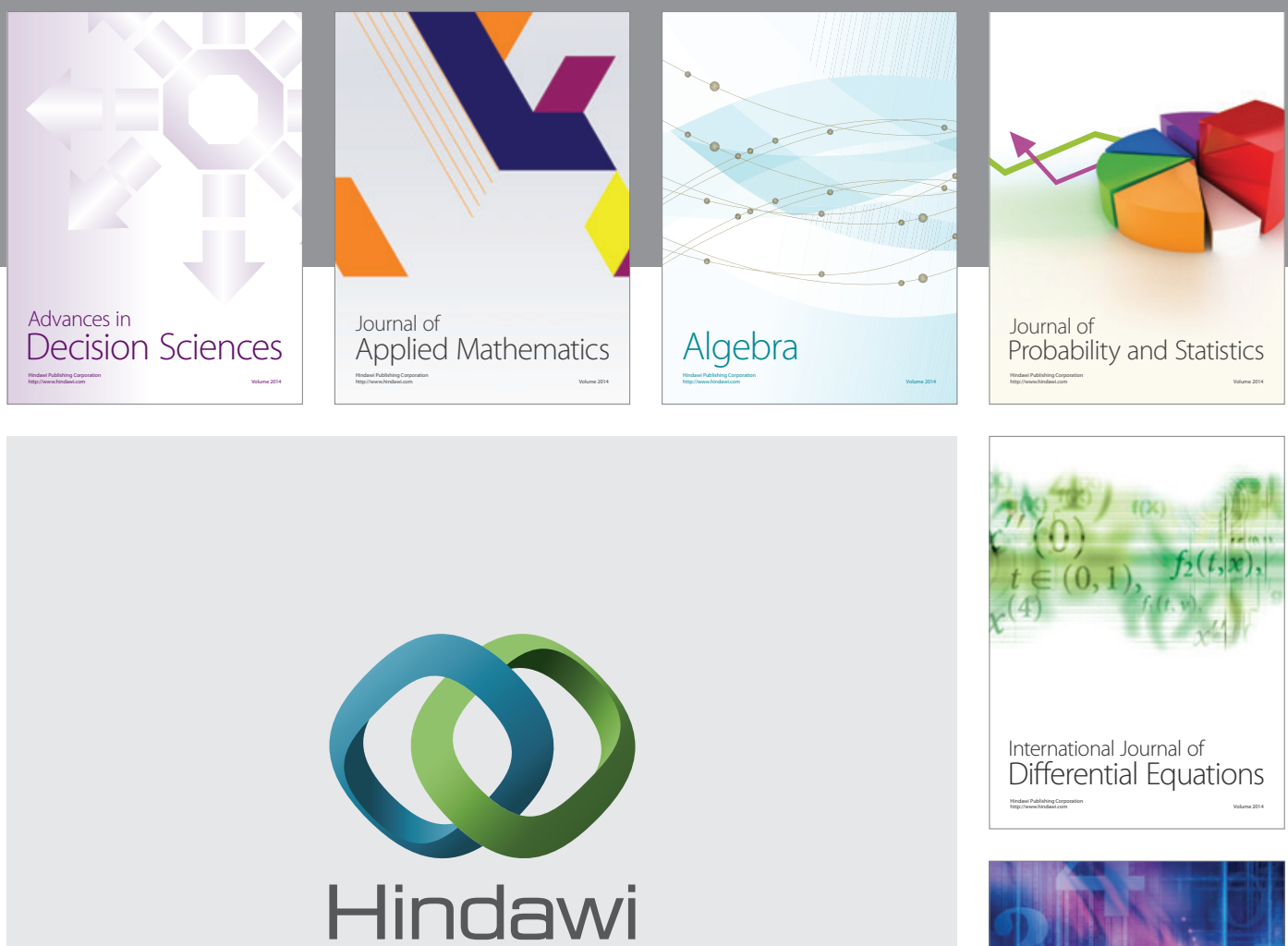

Submit your manuscripts at http://www.hindawi.com
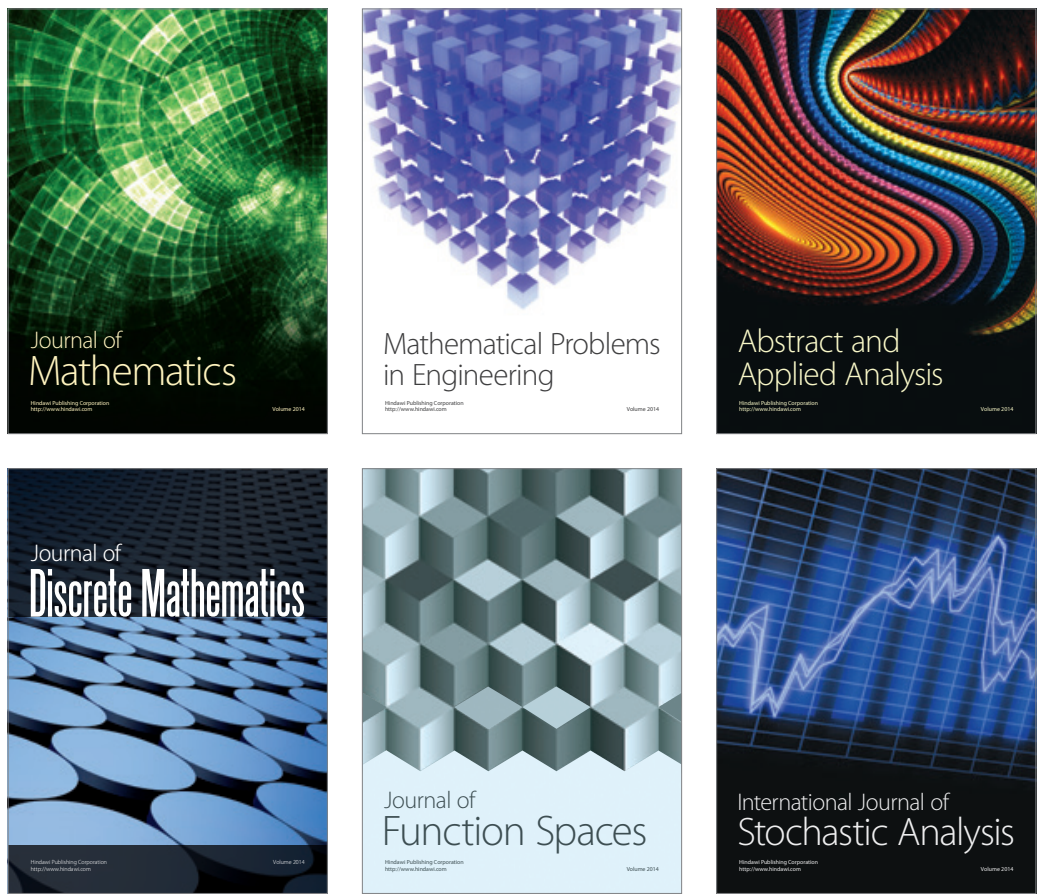

Journal of

Function Spaces

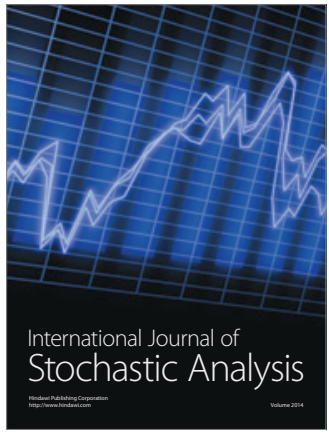

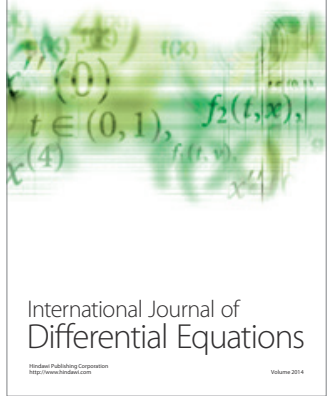
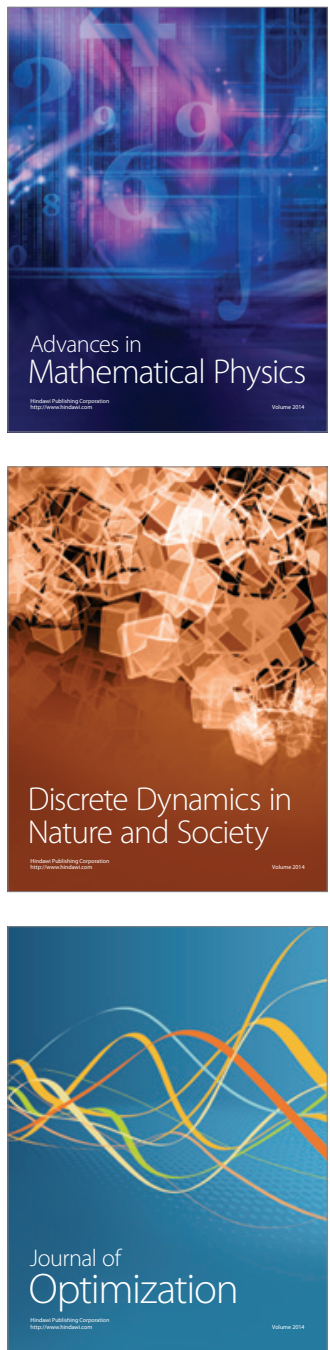